\title{
Suppression of overt attentional capture by salient-but-irrelevant color singletons
}

\author{
Nicholas Gaspelin $^{1}$ • Carly J. Leonard ${ }^{1}$ • Steven J. Luck ${ }^{1}$
}

Published online: 1 November 2016

(C) The Psychonomic Society, Inc. 2016

\begin{abstract}
For more than 2 decades, researchers have debated the nature of cognitive control in the guidance of visual attention. Stimulus-driven theories claim that salient stimuli automatically capture attention, whereas goal-driven theories propose that an individual's attentional control settings determine whether salient stimuli capture attention. In the current study, we tested a hybrid account called the signal suppression hypothesis, which claims that all stimuli automatically generate a salience signal but that this signal can be actively suppressed by top-down attentional mechanisms. Previous behavioral and electrophysiological research has shown that participants can suppress covert shifts of attention to salient-but-irrelevant color singletons. In this study, we used eye-tracking methods to determine whether participants can also suppress overt shifts of attention to irrelevant singletons. We found that under conditions that promote active suppression of the irrelevant singletons, overt attention was less likely to be directed toward the salient distractors than toward nonsalient distractors. This result provides direct evidence that people can suppress salient-but-irrelevant singletons below baseline levels.
\end{abstract}

Keywords Attentional capture · Visual search · Suppression · Eye movements

Electronic supplementary material The online version of this article (doi:10.3758/s13414-016-1209-1) contains supplementary material, which is available to authorized users.

Nicholas Gaspelin

ngaspelin@ucdavis.edu

1 Center for Mind and Brain, University of California, Davis, 267 Cousteau Place, Davis, CA 95618, USA
We often assume that feature singletons, such as uniquely colored objects (called color singletons), can serve as powerful attractors of attention in daily life. For example, lighthouses are frequently painted unnatural colors (e.g., red and white stripes) and equipped with flashing beacons to warn sailors of shipwreck hazards. Similarly, roadwork crews use neon orange traffic cones to warn drivers of potential dangers ahead, such as bumps or potholes. Although these bright, uniquely colored objects seem phenomenologically salient, researchers disagree whether they automatically "capture" visual attention.

Currently, two directly opposed theoretical accounts prevail in the attentional capture literature. According to stimulus-driven theories, salient signals automatically capture visuospatial attention, regardless of a viewer's goals. Support for these theories often comes from studies using the additional singleton paradigm (Theeuwes, 1992). Participants search displays of diamond distractors for a circle target and report the orientation of a line inside the circle. On half of trials, a color singleton appears at a nontarget location. Typically, participants are slower to respond to the target when the singleton is present than when it is absent. This singleton-presence cost is taken as evidence that the task-irrelevant singleton automatically captured spatial attention, slowing attentional allocation to the target.

Stimulus-driven theories garner further support from studies showing that salient stimuli can capture overt eye movements (Theeuwes, Kramer, Hahn, \& Irwin, 1998; Theeuwes, Kramer, Hahn, Irwin, \& Zelinsky, 1999). The underlying assumption of these studies is that participants cannot make an overt eye movement to one location while covertly shifting spatial attention to another location (Hoffman \& Subramaniam, 1995). In other words, an overt shift of attention necessarily implies a preceding shift of covert attention. For example, Theeuwes, De Vries, and Godijn (2003, Experiment 1) adapted the 
additional singleton paradigm for eye tracking. Initial saccades were equally likely to land on the irrelevant color singleton and on the target, suggesting that salient features automatically captured overt and covert attention.

According to the logic of these studies, capture of overt attention necessarily implies the capture of covert attention. However, the absence of overt capture does not imply the absence of covert attentional capture. Indeed, to explain instances where salient stimuli produce no observable overt capture effects, stimulusdriven theorists may invoke the rapid disengagement hypothesis (Theeuwes et al., 2003, Experiment 2; Theeuwes, 2010). According to this account, covert attention initially moves to the most salient item in the display, but the salient item can then be rapidly rejected so that the target is attended with little delay. This hypothesis makes two relevant predictions about overt capture effects. First, covert attention may reject the salient item before overt eye movements are initiated, resulting in large singleton-presence costs on manual response time (RT), but no accompanying oculomotor capture effects (see Theeuwes et al., 2003, Experiment 2). A second prediction is that covert attention may initially be captured by the salient item, but relatively fast eye movements are initiated before the singleton is rejected. This would lead to large oculomotor capture effects on trials with fast saccades and small oculomotor capture effects on trials with slow saccades. For example, van Zoest, Donk, and Theeuwes (2004, Experiment 3) found that, in an additional singleton paradigm, participants made fewer eye movements to targets when saccades were initiated quickly, which they interpreted to support a rapid disengagement model.

In contrast to stimulus-driven theories, goal-driven theories propose that an individual's goals determine whether salient stimuli capture attention (Folk, Remington, \& Johnston, 1992). In other words, visual attention is involuntarily guided to objects that have features matching what the observer is looking for (called an attentional set). For example, a shopper looking for a red can of soup would be involuntarily captured by other red items, such as a red sale sign. However, the shopper would not be captured by nonred items, such as a bright yellow wet floor sign.

To explain apparent instances of stimulus-driven capture, goal-driven theorists note that the target itself is a feature singleton in many of additional singleton paradigm studies, which might lead participants to adopt a broad attentional set for any feature singleton (called singleton-detection mode; Bacon \& Egeth, 1994). Many studies have demonstrated that when participants are instead encouraged to search for a specific shape (called feature-search mode), salient stimuli no longer capture covert attention (Bacon \& Egeth, 1994; Cosman \& Vecera, 2013; Leber \& Egeth, 2006) or overt attention (Leonard \& Luck, 2011; Theeuwes et al., 2003; Wu \& Remington, 2003). For example, Leonard and Luck (2011) had participants perform an additional singleton paradigm, as described above. In Experiment 1, participants searched for a pop-out shape whose exact identity varied randomly trial by trial (e.g., circle among diamonds or diamond among circles). First saccades landed on isoluminant color singletons on a high percentage of trials (15.5\%). In Experiment 2, the exact identity of the target was held constant across trials, allowing participants to use feature-search mode to search for a specific feature. Here, first saccades landed on isoluminant color singletons on significantly fewer trials $(9.8 \%)$.

In summary, stimulus-driven and goal-driven accounts make opposing predictions about when to expect attentional capture. Many researchers have suggested the need for a more coherent account of attentional capture (Awh, Belopolsky, \& Theeuwes, 2012). Resolving this empirical debate with a more nuanced account would greatly benefit our ability to predict eye movements in naturalistic scenes (Henderson, 2003), design more accurate models of visual search (Wolfe, 2007; Wolfe, Cave, \& Franzel, 1989), and understand the development of cognitive control in typical (Gaspelin, MargettJordan, \& Ruthruff, 2014) and atypical (Leonard, Robinson, Hahn, Gold, \& Luck, 2014) populations.

\section{Signal suppression hypothesis}

Sawaki and Luck (2010) proposed a reconciliation to this debate, called the signal suppression hypothesis. According to this hybrid model, a salient stimulus in a visual scene generates a bottom-up "attend-to-me" signal that has the potential to capture attention even if it does not match the observer's goals, consistent with stimulus-driven theories. However, this salience signal can be actively suppressed by means of topdown control processes so that it does not actually capture attention, consistent with top-down theories. This hypothesis differs from stimulus-driven theories in proposing that the salience signal can be suppressed before attention is captured, and it differs from goal-driven theories in proposing that attention will be automatically captured by salient-but-irrelevant stimuli if the salience signal is not suppressed.

According to the signal suppression hypothesis, the active suppression process can be indexed by the distractor positivity $\left(\mathrm{P}_{\mathrm{D}}\right)$ component of the event-related potential (ERP) waveform (Hickey, Di Lollo, \& McDonald, 2009; Sawaki \& Luck, 2010). The initial support for the signal suppression hypothesis came from studies demonstrating a $\mathrm{P}_{\mathrm{D}}$ component to salient stimuli in the absence of behavioral capture (Burra \& Kerzel, 2014; Eimer \& Kiss, 2008; Gaspar, Christie, Prime, Jolicoeur, \& McDonald, 2016; Gaspar \& McDonald, 2014; Jannati, Gaspar, \& McDonald, 2013; Sawaki \& Luck, 2010, 2011). Separate studies have found independent behavioral evidence for suppression of attentional capture by salient items (Gaspelin, Leonard, \& Luck, 2015; Jannati et al., 2013; Moher \& Egeth, 2012; Moher, Lakshmanan, Egeth, \& Ewen, 2014; Vatterott \& Vecera, 2012). For example, 
Gaspelin et al. (2015) developed a capture-probe paradigm in which, on most trials, participants searched displays of shapes for a specific target and made a speeded response to report the location of a dot inside. On occasional probe trials, a letter appeared briefly inside each item of the search array. On these trials, participants were to recall as many letters as possible rather than performing the search task (see also Kim \& Cave, 1995). Under conditions that resulted in no behavioral capture in the visual-search task, participants were actually less likely to report the probe letter at the singleton location than the probe letter at a given nonsingleton location, suggesting the singleton was suppressed below baseline levels of processing.

Despite the aforementioned behavioral and electrophysiological evidence for suppression of attentional allocation to salient singletons, no studies have directly assessed whether humans can actively suppress overt eye movements to salient items. More specifically, no studies of oculomotor control have demonstrated an oculomotor suppression effect - a lower likelihood that salient display items will be the target of the initial eye movement compared to nonsalient display items. This oculomotor evidence is important because previous behavioral suppression effects (e.g., in Gaspelin et al., 2015) may have resulted from suppression at a number of processing stages (e.g., in memory encoding). If suppression can be observed at relatively short saccadic latencies, this will constrain the possible mechanisms that underlie the suppression.

Some studies have demonstrated that oculomotor capture by salient color singletons can be avoided under certain conditions (Leonard \& Luck, 2011; Wu \& Remington, 2003). However, these studies did not find that participants were less likely to fixate salient items than other nontarget display items. Ipata, Gee, Gottlieb, Bisley, and Goldberg (2006), however, did observe such a singleton suppression effect in macaque monkeys. They found that highly trained monkeys made fewer first saccades to singleton distractors $(2 \%)$ than to the average nonsingleton distractor $(8 \%)$. However, the monkeys in this study performed approximately 36,000 trials, and it is unclear if the observed oculomotor suppression would generalize to humans making eye movements in the natural world or to humans performing laboratory tasks for an hour or two. Furthermore, the article did not report the time course of suppression, which is essential for ruling out alternative explanations such as the rapid disengagement hypothesis (e.g., see our Experiment 2). In short, the current evidence for oculomotor suppression is limited, at best.

\section{The Current Study}

In this study, we provide further support the signal suppression hypothesis, demonstrating active suppression of oculomotor capture by salient-but-irrelevant color singletons in humans under typical laboratory conditions. Participants performed a variant of the additional singleton paradigm that was modified for eye tracking (see Fig. 1). To modulate capture by color singletons, we used a similar method to Gaspelin et al. (2015) of manipulating search mode (singleton detection mode vs. feature search mode). We predicted that when the task encouraged participants to search for singletons, the initial saccade on each trial should be more likely to be directed toward the singleton distractor than toward the average nonsingleton distractor (an oculomotor capture effect). In contrast, when the task encouraged participants to attend to the specific shape of the target and avoid singletons, the initial saccade should be less likely to be directed toward the singleton distractor than toward the average nonsingleton distractor (an oculomotor suppression effect). We also investigated the time course of singleton suppression effects to rule out the rapid disengagement hypothesis (see Experiments 2 and 3).

\section{Experiment 1}

Experiment 1 was designed to validate our measures of oculomotor capture under conditions in which irrelevant singletons are known to capture attention. The target itself was a shape singleton (a circle amongst diamonds or a diamond amongst circles). The specific shape of the target varied randomly by trial, forcing participants to use a singleton-

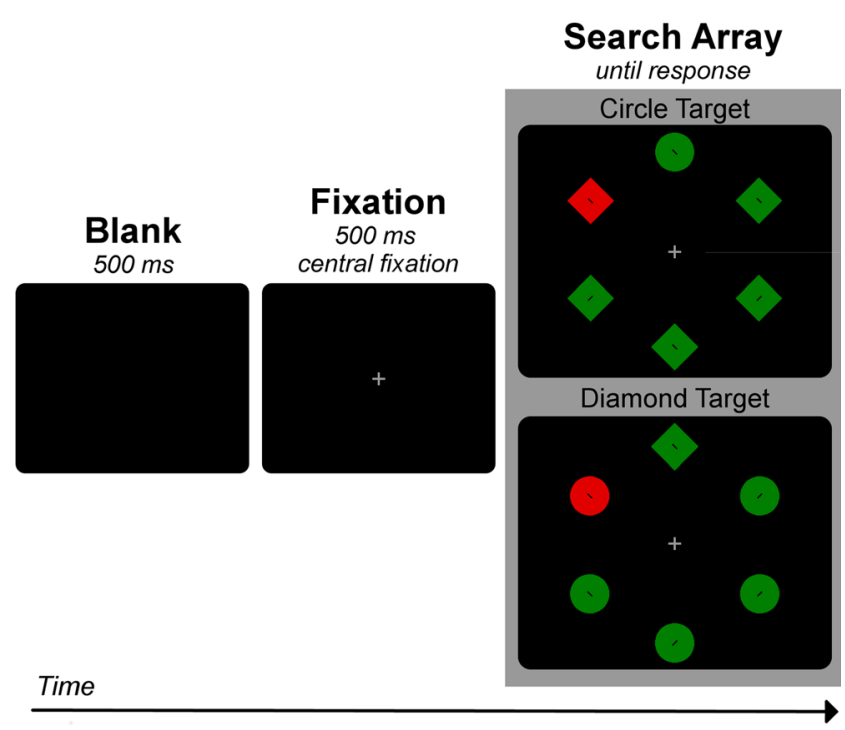

Fig. 1 Stimuli from Experiment 1. The screen was blank for $500 \mathrm{~ms}$ at the beginning of each trial. A fixation cross then appeared, and participants were required to hold their gaze on this point for $500 \mathrm{~ms}$. Finally, the search array appeared and remained visible until the participant responded. Participants searched for the unique shape in the display (a diamond among circles or a circle among diamonds, randomly intermixed). They then reported (via button press) whether the small black line inside the unique shape was tilted left or right. In grayscale versions of this figure, solid lined shapes were one color (e.g., green) while dashed lined shapes were another color (e.g., red). See the online article for color versions of all figures in this paper 
detection mode strategy. This strategy is known to produce large oculomotor capture effects (Leonard \& Luck, 2011; Theeuwes et al., 2003). If our task is a valid measure of oculomotor capture, the first saccade on each trial should be more likely to be directed toward singleton distractors than to the average nonsingleton distractor.

\section{Method}

Participants We chose an a priori sample size of 20 participants per experiment on the basis of our prior behavioral research on singleton suppression (Gaspelin et al., 2015) and previous eye-tracking studies (Leonard \& Luck, 2011). The participants were University of California, Davis students who were paid for participating. One participant in Experiment 1 had abnormally high manual response times (more than 2.5 standard deviations from the group mean) and was replaced. Of the final sample of 20 participants, 16 were female and four were male. The mean age was 21.8 years. All participants had normal color vision and had normal or corrected-to-normal visual acuity.

Apparatus Stimuli were presented using PsychToolbox (Brainard, 1997) on an LCD monitor with a black background that was placed at a viewing distance of $100 \mathrm{~cm}$. A photosensor was used to measure the timing delay of the monitor $(32 \mathrm{~ms})$, and this delay was subtracted from all latency values reported here. An SR Research Eyelink 1000 deskmounted system recorded eye position monocularly from the right eye at $500 \mathrm{~Hz}$.

Stimuli and procedure The stimuli are illustrated in Fig. 1. Each search display contained six items distributed at equal distances around a notional circle with a radius of $4.5^{\circ}$. The individual stimuli were diamonds $\left(0.8^{\circ} \times 0.8^{\circ}\right)$ and circles $\left(0.9^{\circ}\right.$ diameter $)$ drawn in red $\left(23.3 \mathrm{~cd} / \mathrm{m}^{2}, x=.65, y=.34\right)$ or green $\left(23.3 \mathrm{~cd} / \mathrm{m}^{2}, x=.29, y=.63\right)$. Each shape contained a black line subtending $0.30^{\circ} \times 0.05^{\circ}$ that was tilted $45^{\circ}$ to the left or right. A gray fixation cross $\left(23.3 \mathrm{~cd} / \mathrm{m}^{2}, 0.1^{\circ}\right.$ by $\left.0.1^{\circ}\right)$ was continuously visible except during the intertrial interval.

The search target was defined as the unique shape, which was unpredictably a circle among identical diamonds or a diamond among identical circles. The task was to report whether the line inside the target shape tilted to the left or right (by pressing game-pad buttons with the left or right index fingers). Pilot studies indicated that participants could not easily report the orientation of the target line without fixating it. Thus, our task implicitly required eye movements, but we did not explicitly instruct participants to move their eyes toward the target. Target location and target line tilt varied randomly. All items were a single color on $50 \%$ of trials (red for half of the participants and green for the others), and one item was drawn in the other color on the remaining trials. The location of this color singleton distractor was random, except that it was never the target location. Participants were told this and were encouraged to ignore the color singleton.

Trials began with the presentation of a blank screen for $500 \mathrm{~ms}$. This was followed by a fixation screen containing only the fixation point; this screen remained visible until the participant maintained fixation within a $1.5^{\circ}$ radius of the fixation point for $500 \mathrm{~ms}$. The search array then appeared and remained visible until response. If participants took too long to respond (more than $3,000 \mathrm{~ms}$ ), a time-out display appeared with the text "Too Slow" for $500 \mathrm{~ms}$. If the response was incorrect, a $200-\mathrm{Hz}$ tone sounded for $500 \mathrm{~ms}$. The blank screen for the next trial then appeared immediately.

Participants practiced the search task for two blocks of 64 trials. The main experiment consisted of six blocks of 64 trials, yielding 384 trials, 192 with and 192 without an irrelevant singleton. Participants received block-by-block feedback on mean response time (RT) and accuracy. At the beginning of each block, the eye tracker was calibrated using a 9-point calibration technique. During the main task, the eye tracker was recalibrated if a participant failed to fixate the central cross for more than 8 seconds at the beginning of a trial.

Analysis The onset of a saccade was defined using a minimum eye velocity threshold of $30^{\circ}$ per second and a minimum acceleration threshold of $9,500^{\circ}$ per second. To classify the landing position of the first saccade on each trial, an annulus was defined that was centered on the fixation cross, with an inner radius of $1.5^{\circ}$ and an outer radius of $7.5^{\circ}$. First saccades were defined as the first eye movement landing inside the annulus. The landing position was then classified by selecting the nearest search item. This technique effectively created wedge-shaped interest areas around each search item. Saccadic latency was measured as the start time of the first saccade that landed in the annulus. ${ }^{1}$

We excluded trials with abnormal manual response times (less than $200 \mathrm{~ms}$ or greater than $2,000 \mathrm{~ms}$, accounting for $0.6 \%$ of trials), trials in which participants made no eye movement (1.7\% of trials), and trials with abnormal saccade latencies (less than $50 \mathrm{~ms}$ or greater than $1,000 \mathrm{~ms}$, accounting for $3.6 \%$ of trials). Additionally, we excluded trials with manual response errors $(2.7 \%)$ from all analyses except manual response error analyses. Altogether, $6.8 \%$ of trials were excluded. In all analyses of variance (ANOVAs) reported in this article, we report the Greenhouse-Geisser corrected $p$ value.

\footnotetext{
${ }^{1}$ The basic pattern of results in every experiment of this article was the same with or without the interest area annulus.
} 


\section{Results}

\section{Manual responses}

As shown in Fig. 2a, manual responses in the search task were slower when the color singleton was present $(852 \mathrm{~ms})$ than when it was absent $(801 \mathrm{~ms})$ - a 51-ms singleton-presence cost, $t(19)=$ $7.29, p<.001$. These results confirm that this task effectively yielded strong attentional capture by the irrelevant color singleton. Error rates were similar for singleton-absent trials $(2.7 \%)$ and singleton-present trials $(2.7 \%), t(19)<1, p=.821$.

Some goal-driven researchers have argued that singletonpresence costs may reflect nonspatial filtering costs (Folk \& Remington, 1998) in which the appearance of the singleton disrupts processing without attracting spatial attention to its location. To argue against this account, previous researchers have shown that singleton interference increases when the singleton appears closer to the target (Gaspar \& McDonald, 2014; Mounts, 2000). As shown in Fig. 2b, manual responses were indeed much slower when the singleton appeared at the $+/-60^{\circ}$ angular distance from the target compared to when the singleton appeared at the $+/-120^{\circ}$ or $180^{\circ}$ angular distances. To formally analyze this trend, we conducted a one-way repeated-measures ANOVA comparing mean manual RTs at each singleton angular distance $\left(60^{\circ}, 120^{\circ}, 180^{\circ},-120^{\circ}\right.$, and $\left.-60^{\circ}\right)$ on singleton-present trials. This yielded a main effect of singleton angular distance, $F(4,76)=21.94, p<.001, \eta_{\mathrm{p}}{ }^{2}=$ .536 , indicating that manual RT declines as the angular distance between the target and the irrelevant singleton increases.

\section{First saccade destination}

If the singleton distractor captures the eyes, first saccades should be more likely to move to the singleton distractor than to the average nonsingleton distractor. As shown in Fig. 2c, we found that first saccades were nearly twice as likely to be directed toward the singleton distractor $(16 \%)$ as the average nonsingleton distractor $(9 \%)$ when the color singleton was present (see the part of Fig. 2c labeled oculomotor capture effect). This $7 \%$ singleton enhancement effect was statistically significant, $t(19)=2.58, p=.02$, and it demonstrates that our oculomotor capture task provides a sensitive means of assessing the capture of overt attention.

We also examined how the presence or absence of a singleton impacted first saccades to the target and nonsingleton distractor items. If the singleton captures the eyes, then the first saccade should be less likely to land on the target location. Indeed, first saccades were less likely to land in the target region when the singleton was present than when the singleton was absent (see the leftmost portion of Fig. 2c). First saccades to the nonsingleton distractor locations were not influenced by the presence or absence of the color singleton.
These effects of singleton presence on first saccades to the target and average nonsingleton-distractor locations were analyzed in a two-way ANOVA with factors of singleton presence and item type (target vs. average nonsingleton distractor). This ANOVA excluded data from the singleton location itself, which was necessary to obtain a balanced factorial design given that there was no singleton distractor location in singleton-absent trials. First saccades were more likely to be directed toward the target location (54\%) than toward nonsingleton distractor locations (12\%), which led to a significant main effect of item type, $F(1,19)=103.69, p<.001$, $\eta_{\mathrm{p}}{ }^{2}=.845$. This $42 \%$ effect presumably reflects the attentional priority of the target. The finding that first saccades were less likely to be directed toward the target on singleton-present trials than on singleton-absent trials led to both a significant main effect of singleton presence, $F(1,19)=22.47, p<.001$, $\eta_{\mathrm{p}}{ }^{2}=.542$, and a significant interaction between singleton presence and search item type, $F(1,19)=27.32, p<.001$, $\eta_{\mathrm{p}}{ }^{2}=.590$. Follow-up $t$ tests indicated that the difference between singleton-present and singleton-absent trials was significant at the target location $(8 \%), t(19)=5.24, p<.001$, but was not significant at the nonsingleton-distractor locations $(0 \%), t(19)>1, p=.43$. These results provide additional evidence that gaze was captured by the irrelevant singleton, thereby decreasing the probability that the initial saccade would be directed toward the target.

\section{Oculomotor capture effects by singleton angular distance}

To provide a more direct picture of the distribution of first saccade positions, we created heat maps showing the spatial distribution of first saccades. The data were rotated so that the target location appears at the 12 o'clock position. As shown in Fig. 2d, participants were generally more likely to fixate the location of the singleton distractor than to fixate that same location on singleton-absent trials. Formal statistical analyses of these heat maps are presented in supplementary online materials.

\section{Saccadic latencies}

We also analyzed saccadic latencies as a function of saccade landing position and singleton presence. One participant never fixated the color singleton and was removed from this analysis. As shown in Table 1, saccadic latencies were faster for first saccades that landed on the singleton distractor $(215 \mathrm{~ms})$ than for first saccades that landed on a nonsingleton distractor (229 ms), $t(18)=2.44, p=.03$, or on the target $(263 \mathrm{~ms}), t(18)$ $=5.93, p<.001$, replicating previous research (Theeuwes et al., 2003; Theeuwes et al., 1999). There was a nonsignificant trend for first saccades that landed on the target to be faster when the singleton was present $(256 \mathrm{~ms})$ rather than absent (263 ms), $t(18)=1.87, p=.08$, consistent with previous studies (van Zoest et al., 2004). 


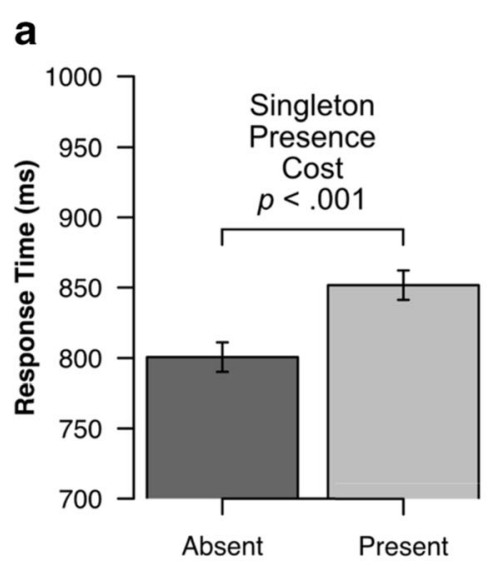

Singleton Presence

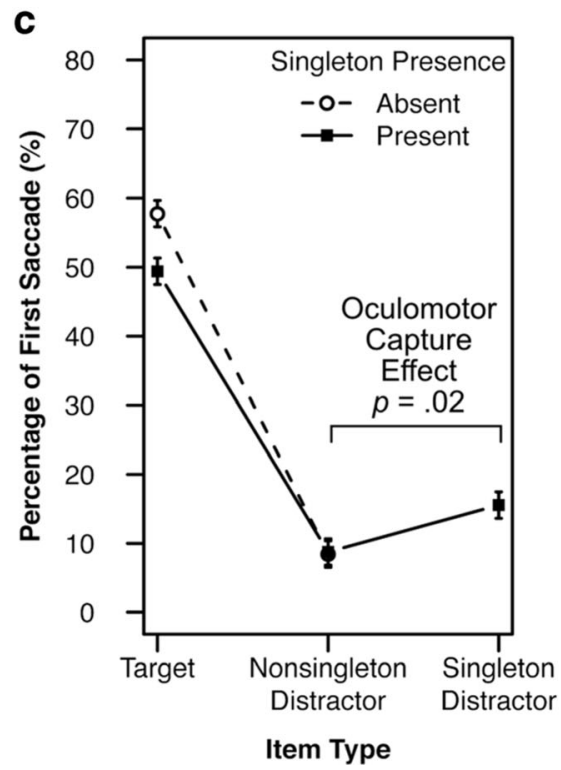

Fig. 2 Results from Experiment 1. (a) Manual response time as a function of singleton presence. (b) Manual response times as a function of the angular distance between the irrelevant singleton and the target. (c) Percentage of first saccades that landed on a given stimulus type (target, nonsingleton distractor, or singleton) on trials with and without a color singleton. For the nonsingleton distractors, this is the mean per distractor, not the mean summed across the several distractors. (d) Heat maps of first-saccade landing position for each singleton position relative to the

\section{Discussion}

Experiment 1 demonstrated the validity and sensitivity of our oculomotor capture task. We observed large behavioral and oculomotor capture effects by task-irrelevant color singletons. Of particular importance, first saccades were more likely to be directed toward singleton items than nonsingleton items, indicating that overt attention was captured. These findings demonstrate that our methods can replicate the oculomotor capture effects observed in previous studies (Theeuwes et al., 2003; Theeuwes et al., 1998; Theeuwes et al., 1999), which was necessary to target location. The data from each trial have been rotated as if the top location contained the target. Arrows point to the singleton location. All heat maps were generated using a Gaussian filter with a smoothing kernel of $0.3^{\circ}$ of visual angle. All error bars shown here and in subsequent figures represent within-subject $95 \%$ confidence intervals (Loftus \& Masson, 1994). See the online article for a color version of this figure. (Color figure online)

Table 1 Mean saccadic latency (ms) by first saccade landing position for Experiment 1

\begin{tabular}{llll}
\hline & Target & $\begin{array}{l}\text { Nonsingleton } \\
\text { Distractor }\end{array}$ & $\begin{array}{l}\text { Singleton } \\
\text { Distractor }\end{array}$ \\
\hline Singleton Present & $263(53.9)$ & $229(45.7)$ & $215(27.8)$ \\
Singleton Absent & $256(46.5)$ & $223(36.0)$ & - \\
\hline
\end{tabular}

Note. Standard deviations are shown in parentheses. 
establish the validity of our approach for assessing oculomotor suppression in the following experiments.

\section{Experiment 2}

In Experiment 2, we made relatively subtle changes to the stimuli to encourage participants to search for a specific feature value and discourage singleton detection mode. Each theory makes a unique prediction about the probability that the first saccade will land on the singleton compared to a given nonsingleton distractor under these conditions. Goal-driven theories assume no special processing of feature singletons per se. Thus, previous goal-driven studies lead to the prediction of an equivalent probability that the first saccade will be directed toward the singleton or toward a given nonsingleton distractor (Folk et al., 1992). Stimulus-driven theories propose singletons should still attract the eyes, leading to an increased probability that the first saccade will be directed to the singleton (an oculomotor capture effect). The signal suppression hypothesis proposes that singletons will be actively suppressed, leading to a decreased probability that the first saccade will be directed toward the singleton relative to a given nonsingleton distractor (an oculomotor suppression effect).

The rapid disengagement hypothesis could also potentially explain a decreased probability that the first saccade was directed toward the singleton. This hypothesis proposes that covert attention is captured by the salient item but can rapidly disengage from it, eliminating any oculomotor capture effects. We tested several predictions of this hypothesis. First, a shift of covert attention to the color singleton will take some time (Theeuwes, 2010). Because there is no delay between the singleton and search display in this study, rapid disengagement predicts an observable behavioral costs on manual RT (Chen \& Mordkoff, 2007; Theeuwes, Atchley, \& Kramer, 2000; Theeuwes et al., 2003, Experiment 2). The signal suppression hypothesis, however, proposes that items are suppressed without an initial shift of attention, which should lead to no observable behavioral costs on manual RT.

A second prediction of the rapid disengagement hypothesis is that gaze will sometimes shift before the singleton has been rejected, and on these trials the first saccade will likely be directed toward the color singleton (van Zoest et al., 2004). Thus, according to this account, the fastest saccades should be more likely to land on the color singleton distractor than the average nonsingleton distractors (i.e., an oculomotor capture effect as in Experiment 1). By contrast, the signal suppression hypothesis posits no initial shift of attention to the color singleton, and no overt capture effects should be present even on trials with the fastest saccade latencies. We performed two converging tests to investigate this possibility: (1) We investigated the landing position of the fastest quartile of saccades, and (2) we investigated average eye position at each moment in time, which does not require considering saccades as temporally discrete events and does not require dividing trials into discrete categories on the basis of saccadic reaction time.

\section{Method}

The methods were identical to those of Experiment 1 with the following changes. First, a new set of 20 students participated (16 females and four males; mean age: 21.1 years). Second, search displays were modified to encourage participants to search for a specific shape rather than searching for singletons (see Fig. 3). Specifically, the target was always the diamond for half of the participants and always the circle for the other half. To prevent the target from "popping out," distractor shapes were randomly generated as squares $\left(0.8^{\circ}\right.$ in width and height), hexagons ( $0.8^{\circ}$ in width and height), and the unselected target shape (circle in the diamond-target condition and diamond in the circle-target condition), with the constraint that no distractor shape could be used in the same search display more than twice. By keeping the target shape constant for a given participant, and by presenting it among a heterogeneous set of distractor shapes, this experiment promoted feature search mode and eliminated any incentives to intentionally search for singletons (Bacon \& Egeth, 1994).

We excluded trials with abnormal manual response times (1.4\%; less than $200 \mathrm{~ms}$ or greater than 2,000 ms), trials in which participants made no eye movement $(0.8 \%)$, and trials with abnormal saccade latencies $(2.6 \%$; less than $50 \mathrm{~ms}$ or greater than 1,000 ms) from all analyses. Additionally, we excluded trials with manual response errors $(2.6 \%)$ from all analyses except manual response error analyses. Altogether, $6.5 \%$ of trials were excluded.

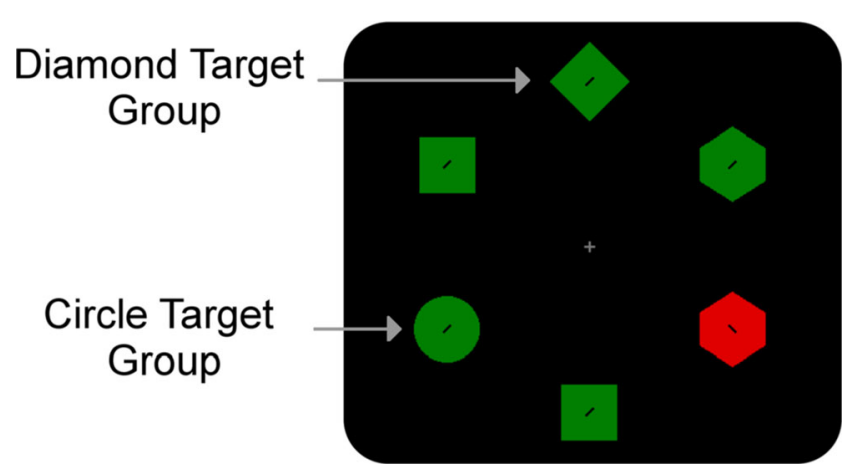

Fig. 3 Example search array from Experiment 2. Each participant was assigned to a target group (circle or diamond), which remained constant throughout the experiment for that participant. Participants were instructed to report the orientation of the line inside the target. Distractors were heterogeneous shapes to prevent the target from popping out. In grayscale versions of this figure, solid lined shapes were one color (e.g., green) while dashed lined shapes were another color (e.g., red). See the online article for a color version of this figure 


\section{Results}

\section{Manual responses}

As shown in Fig. 4a, manual responses in the search task were actually slightly faster when the color singleton was present $(840 \mathrm{~ms})$ than when it was absent $(853 \mathrm{~ms})$, although this $13-$ $\mathrm{ms}$ singleton presence benefit was only marginally significant, $t(19)=1.90, p=.07$. This kind of reversed capture effect has been observed in previous experiments using feature-search tasks (e.g., Gaspelin et al., 2015), and it is consistent with a decreased attentional priority at the singleton location, which effectively reduces the set size. There was also a trend for participants also committed more errors on singleton-absent trials $(3.0 \%)$ than on singleton-present trials $(2.5 \%), t(19)=1.86, p=.08$.

We also assessed whether RTs differed as a function of the angular distance between the target and the singleton, using the same ANOVA design as in Experiment 1. As shown in Fig. 4b, there was no hint of longer mean RTs at the closer distances, and the main effect of angular distance was not significant, $F(4,76)<1, p=.647, \eta_{\mathrm{p}}^{2}=.029$. These results provide additional evidence that the color singleton did not capture covert attention, as predicted by stimulus-driven models such as the rapid disengagement hypothesis.

\section{First saccade destination}

If the color singleton is actively suppressed, the first saccade should be less likely to land on the singleton distractor than to land on the average nonsingleton distractor. As shown in Fig. 4c, first saccades were much less likely to be directed to singleton distractors $(4 \%)$ than to the average nonsingleton distractor $(10 \%), t(19)=7.07, p<.001$ (see the part of Fig. $4 \mathrm{c}$ labeled oculomotor suppression effect). This $6 \%$ suppression effect is consistent with the signal suppression hypothesis but inconsistent with purely goal-driven or stimulus-driven accounts.

We also analyzed the effect of singleton presence on first saccades to the target and nonsingleton distractors in a two-way ANOVA (excluding the singleton location, as in Experiment 1). This ANOVA yielded an effect of item type, $F(1,19)=101.92, p$ $<.001, \eta_{\mathrm{p}}{ }^{2}=.843$, indicating that first saccades were more likely to be directed toward the target (55\%) than toward the average nonsingleton distractor $(10 \%)$. This $45 \%$ effect presumably reflects the top-down attentional priority of the target. The ANOVA also yielded a marginally significant main effect of singleton presence, $F(1,19)=3.573, p=.07, \eta_{\mathrm{p}}{ }^{2}=.158$, reflecting the finding that first saccades were slightly more likely to be directed to targets and nonsingleton distractors on singleton-present trials (33\%) than on singleton-absent trials $(31 \%)$. The interaction between singleton presence and item type was not significant, $F(1,19)<1, p=.985, \eta_{\mathrm{p}}{ }^{2}=.001$, indicating that the presence of a singleton had no differential effect on first saccades to the target versus the nonsingleton distractors.
Suppression effects by singleton angular distance

Figure $4 d$ uses heat maps to provide a more detailed picture of the distribution of first saccade landing positions. Participants were generally less likely to fixate the location of a singleton distractor than to fixate the same location on singleton-absent trials. For example, very few first saccades landed at the $-60^{\circ}$ location when this location contained the singleton than when the singleton at one of the other locations. Formal statistical analyses of these heat maps are presented in supplementary online materials. Here, we briefly note that suppression effects were significant at each singleton angular distance $(p<.01)$.

\section{Saccadic latencies}

We analyzed saccadic latencies as a function of saccade destination and singleton presence. One participant never fixated the color singleton and was removed from the analysis. As shown in Table 2, saccadic latencies were shorter for first saccades that landed on the singleton distractor $(194 \mathrm{~ms})$ than for first saccades that landed on the nonsingleton distractor $(218 \mathrm{~ms}), t(18)=2.62, p=.02$, or target $(245 \mathrm{~ms}), t(18)=$ $5.00, p<.001$. At first glance, these results may seem to support rapid disengagement. However, such differences in saccadic latencies could also be explained by the signal suppression hypothesis: Top-down control may wax and wane from trial-to-trial, and gaze will be captured by the singleton primarily on trials with poor top-down control. As shown by research using the antisaccade task (reviewed by Hutton \& Ettinger, 2006), saccadic latencies are likely to be fastest on trials with poor top-down control, and this would lead to faster saccadic RTs when gaze is directed to the singleton.

Note that saccade latencies for first fixations to the target were not significantly different when the color singleton was present versus absent, $t(18)=1.46, p=.16$. In fact, the nonsignificant trend went in the opposite direction predicted by rapid disengagement models (van Zoest et al., 2004), with participants being slightly faster when the singleton was present $(245 \mathrm{~ms})$ than when it was absent $(250 \mathrm{~ms})$.

\section{Suppression effects for the fastest quartile of saccadic latencies}

To directly assess the rapid disengagement hypothesis, we classified each participant's saccadic latencies into four quartiles: fastest saccade trials, fast saccade trials, slow saccade trials, and slowest saccade trials. ${ }^{2}$ We then compared oculomotor suppression effects in each quartile, defined as the difference in the

\footnotetext{
${ }^{2}$ We chose to divide the data into four quantiles (rather than eight or 10) because it maintained reasonably high statistical power (48 singletonpresent trials per quantile) while allowing us to observe a wide range of mean saccadic latencies.
} 


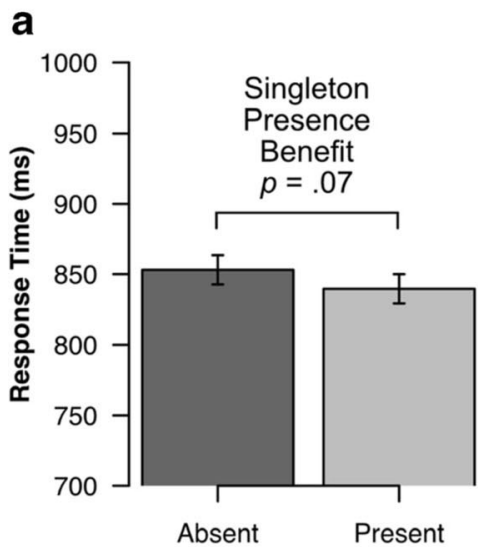

Singleton Presence

C

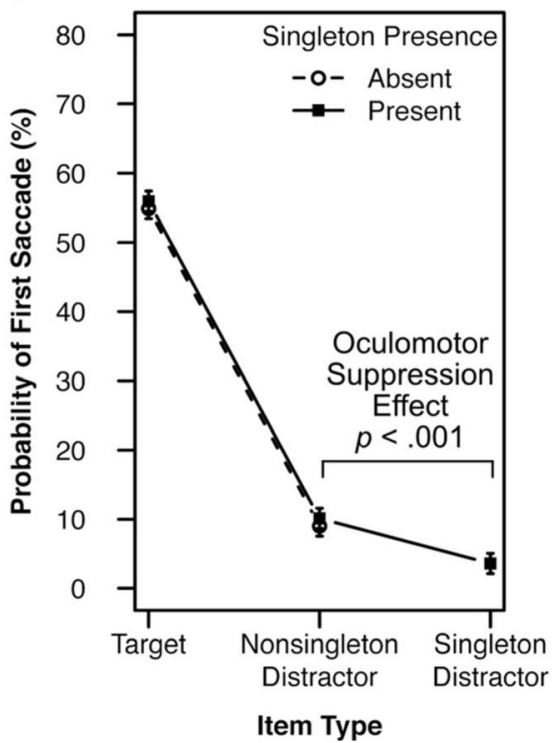

Fig. 4 Results from Experiment 2. (a) Manual response time as a function of singleton presence. (b) Manual response time as a function of the angular distance between the irrelevant singleton and the target. (c) Percentage of first saccades that landed on a given stimulus type (target, nonsingleton distractor, or singleton). (d) Heat maps of first saccade

proportion of first saccades directed to the singleton location versus the average nonsingleton location on singleton-present trials (see Table 3). The rapid disengagement hypothesis predicts that in the fastest quartile, first saccades should be more likely to land on the singleton distractor than on the average nonsingleton distractor (van Zoest et al., 2004). The signal suppression hypothesis, however, posits no initial shift of attention to the color singleton, and no singleton capture effect should be present even on fast-saccade trials. As shown in Table 3, we found approximately equivalent oculomotor suppression effects at all four quartiles. Critically, in the fastest quartile (mean latency: $174 \mathrm{~ms}$ ), first saccades were significantly less likely to land on the singleton distractor than on the average nonsingleton distractor, $t(19)=$ landing position for each singleton position relative to the target location. The data from each trial have been rotated as if the top location contained the target. Arrows point to the singleton location. See the online article for a color version of this figure. (Color figure online)

$3.75, p=.001$. Suppression effects were significant in each of the other quartiles as well $(p<.001)$. We also compared the suppression effects across quartiles in a one-way ANOVA, and

Table 2 Mean saccadic latency (ms) by first saccade landing position for Experiment 2

\begin{tabular}{llll}
\hline & Target & $\begin{array}{l}\text { Nonsingleton } \\
\text { Distractor }\end{array}$ & $\begin{array}{l}\text { Singleton } \\
\text { Distractor }\end{array}$ \\
\hline Singleton Present & $245(42.1)$ & $218(36.5)$ & $194(35.4)$ \\
Singleton Absent & $250(48.9)$ & $218(40.3)$ & - \\
\hline
\end{tabular}

Note. Standard deviations are shown in parentheses. 
Table 3 Mean Saccadic latency (ms), likelihood of initially fixating the nonsingleton and singleton distractors, and suppression effects for each saccadic latency quartile in Experiment 2

\begin{tabular}{llccc}
\hline Quartile & Mean Saccadic Latency & Nonsingleton Distractor & Singleton Distractor & Suppression Effect \\
\hline Fastest & 174 & $14 \%$ & $7 \%$ & $7 \%$ \\
Fast & 209 & $11 \%$ & $3 \%$ & $8 \%$ \\
Slow & 239 & $9 \%$ & $2 \%$ & $7 \%$ \\
Slowest & 312 & $7 \%$ & $2 \%$ & $5 \%$ \\
\hline
\end{tabular}

Note. Suppression effects were calculated as the percentage of saccades landing on the average nonsingleton distractor minus the percentage of saccades landing on the singleton distractor on singleton-present trials.

we found that the magnitude of the suppression did not significantly differ by quartile, $F(3,57)<1, p=.433, \eta_{\mathrm{p}}{ }^{2}=.043$. These results directly contradict the prediction of the rapid disengagement hypothesis that the singleton should capture gaze when the saccadic latencies are fast. Instead, these results are consistent with the signal suppression hypothesis.

\section{Continuous gaze position}

To further test the rapid disengagement hypothesis, we also examined gaze position at each sample point, eliminating the need to divide the data into quantiles and to identify saccades and fixations. The data are shown in Fig. 5. Each trial was normalized so that the target appeared at the 12 o'clock position. Trials were then separated on the basis of the angular distance between the singleton and the target. We then plotted gaze position at each sample point for the first $400 \mathrm{~ms}$ of each trial. The rapid disengagement hypothesis predicts that gaze should be more likely to be directed toward the color singleton than toward the average nonsingleton distractor early in the trial (an oculomotor capture effect), and only later in the trial should gaze be more likely to be directed toward the average nonsingleton distractor than toward the color singleton.

Animated plots for each angular distance can be found online as supplementary material (and make the pattern of data extremely clear). Figure 5a shows representative plots from the $-60^{\circ}$ angular distance at selected time points. Gaze locations initially start near the fixation point and then become distributed among the display items. Before the 200-ms time point, the number of samples within the singleton region was no greater than the number of samples within the nonsingleton distractor regions. As the trial progressed, however, gaze positions become more selective, with most samples located within the target region, fewer within the nonsingleton distractor regions, and even fewer within the singleton distractor region. The key observation is that, in contrast with the prediction of the rapid disengagement hypothesis, gaze samples early in the trial were not more likely to be directed toward the color singleton than toward the other locations.

To formally analyze these data, we determined the closest object to the current gaze position at each sample point (every
$2 \mathrm{~ms}$ ). This effectively divided the entire search display into six pie-slice-shaped regions of interest. We then computed the proportion of gaze positions in each region (target, singleton distractor, and average of the nonsingleton distractors) for each time sample. For simplicity, we averaged the data over all singleton angular distances, as shown in Fig. 5b. We then ran $t$ tests comparing the proportion of samples in the singleton region versus the proportion of samples in the nonsingleton regions at each time point. Time points with a significant difference (uncorrected for multiple comparisons) are shaded in gray in Fig. 5b. As the figure shows, samples were equally likely to be in the target, singleton distractor, and nonsingleton distractor regions until approximately $200 \mathrm{~ms}$, and at no time point were samples more likely to occur in the singleton region than in the average of the nonsingleton distractor regions. Gaze samples were significantly more likely to be located within the average of the nonsingleton distractor regions than within the singleton region from $208 \mathrm{~ms}$ to $400 \mathrm{~ms}$ after the search array onset.

Because uncorrected $p$ values were used in these analyses, it was necessary to provide an additional analysis using an approach that can control the Type I error rate. We therefore used a nonparametric permutation-based analysis that was originally developed to assess the time course of differences between event-related potential waveforms (Groppe, Urbach, \& Kutas, 2011; Maris \& Oostenveld, 2007) and has been adapted to assess the time course of differences in gaze position (Oakes, Baumgartner, Barrett, Messenger, \& Luck, 2013). ${ }^{3}$ We conducted 1,000 random permutations of the data, and the observed run length of 91 consecutive samples was well beyond the 95th percentile of the null distribution. Thus, the observed period of differences between the singleton

\footnotetext{
${ }^{3}$ This approach assumes that gaze position is relatively stable from sample to sample, so true differences between conditions will typically consist of long runs of sample periods in which an uncorrected significant difference is present. The approach then asks whether the observed length of a run of significant $t$ values is greater than one would expect by chance. A permutation analysis is conducted in which the location labels are randomly permuted trial by trial to obtain an empirical null distribution of the number of consecutive significant differences that would be expected by chance. If the observed number of consecutive significant differences is beyond the 95th percentile of this null distribution, then that period of differences is considered statistically significant.
} 
a

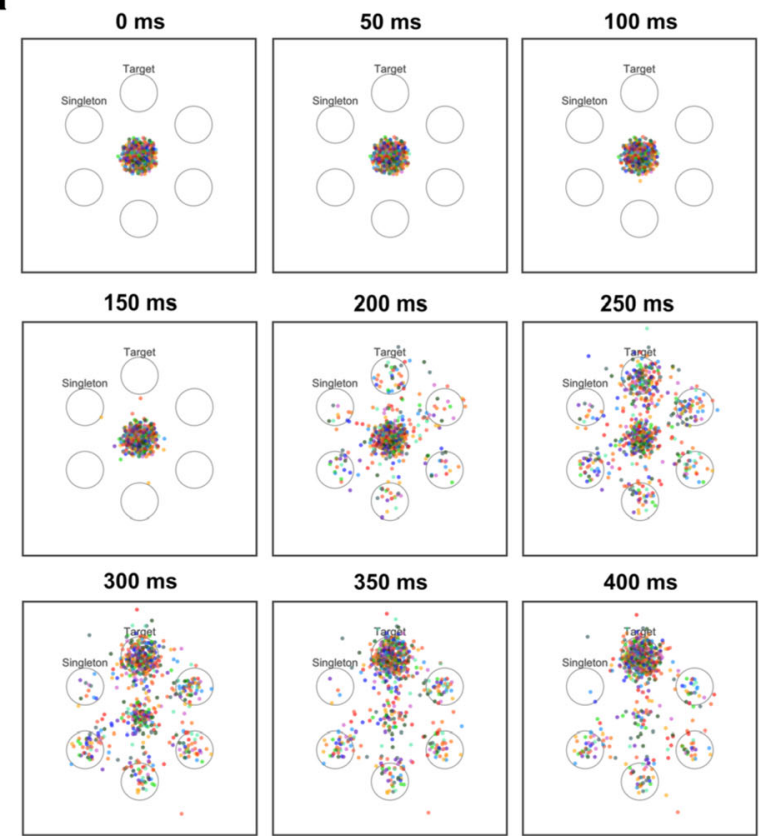

Fig. 5 Gaze position as a function of time relative to stimulus onset in Experiment 2. An animated plot can be found online as supplementary material. (a) Scatterplots of gaze position at selected time points from trials in which the singleton was $60^{\circ}$ counterclockwise from the target (with the data rotated so that the target appears at the 12 o'clock position). Each dot represents a participant's eye position at that moment on a single trial. At each time point from $200 \mathrm{~ms}$ onward, fewer gaze samples were located in the irrelevant singleton location than in the average

region and the average of the nonsingleton distractor regions was statistically significant.

We have additionally included this same gaze position analysis for Experiment 1 in the supplementary online material. There, we find oculomotor capture instead of oculomotor suppression, demonstrating the general validity of our approach.

Altogether, the results for Experiment 2 provide no support for the hypothesis that gaze was captured by the singleton distractor early in the trial. Instead, the singleton location was suppressed as soon as nonrandom eye movements were being made.

\section{Discussion}

The results of Experiment 2 provide evidence that when a task is designed in a manner to discourage singleton detection mode, eye movements to singletons are actively suppressed below baseline levels. This suppression effect was directly predicted by the signal suppression account but not by traditional goal-driven or stimulusdriven theories. Furthermore, there was no evidence that this suppression effect reflected an initial capture of covert attention by the salient item, followed by rapid disengagement. b

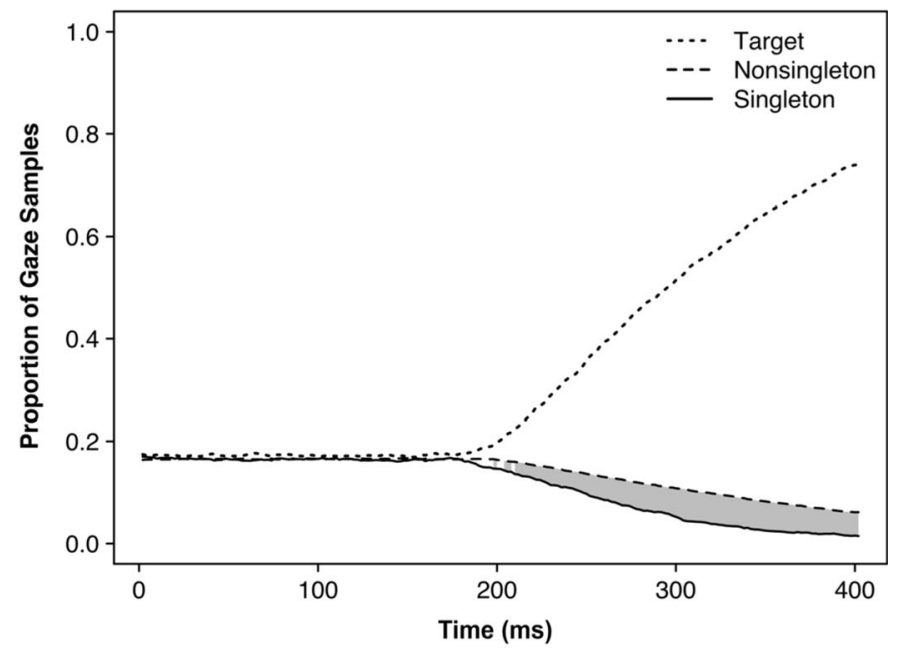

nonsingleton distractor location. (b) Data from (a) reformatted as the proportion of gaze samples in the three object interest regions (target, singleton distractor, average nonsingleton distractor) at each time point. To simplify the analyses, we averaged across every singleton angular distance. Shaded gray regions represent regions where the suppression effect (nonsingleton minus singleton) is statistically significant $(p<.05)$. (Color figure online)

\section{Experiment 3}

An alternative explanation for the results of Experiment 2 is that all of the eye movements were too slow to observe an initial attentional capture by the singleton distractor. Two factors might have slowed saccades in that experiment. First, participants were required to hold their gaze at the central fixation point for $500 \mathrm{~ms}$ before the search array appeared. This long fixation requirement might have encouraged slow, highly controlled eye movements. Second, the fixation point remained visible when the search array appeared, which is known to lead to slower saccadic latencies (Fischer \& Breitmeyer, 1987; Reuter-Lorenz, Hughes, \& Fendrich, 1991).

In Experiment 3, we replicated Experiment 2, but with two changes designed to encourage faster saccade latencies. First, we used a more relaxed central fixation requirement $(100 \mathrm{~ms})$. Second, the central fixation point disappeared at the onset of the search array. According to the rapid disengagement hypothesis, the faster saccadic reaction times resulting from these changes in Experiment 3 should eliminate the oculomotor suppression effects that we observed in Experiment 2 because the time to enact top-down suppression is reduced. According to the signal suppression hypothesis, however, oculomotor suppression effects should still be robust. 


\section{Method}

The methods were identical to those of Experiment 2 except for the following changes. First, a new set of 20 students participated (14 females and six males; mean age: 21.1 years). Second, the search array appeared as soon as the participants fixated the central fixation point for $100 \mathrm{~ms}$. Third, the fixation cross disappeared from the center of the screen when the search array appeared.

As in Experiment 2, we excluded trials with abnormal manual response times $(0.7 \%$; less than $200 \mathrm{~ms}$ or greater than $2,000 \mathrm{~ms}$ ), trials in which participants made no eye movements $(0.1 \%)$, and trials with abnormal saccade latencies (2.2\%; less than $50 \mathrm{~ms}$ or greater than 1,000 ms) from all analyses. Additionally, we excluded trials with manual response errors $(2.8 \%)$ from all analyses except manual response error analyses. Altogether, we excluded $5.5 \%$ of trials.

\section{Results}

\section{Manual responses}

The manual response results (see Fig. 6a) were quite similar to those from Experiment 2. Responses were faster when the color singleton was present $(820 \mathrm{~ms})$ than when it was absent ( $844 \mathrm{~ms}$ ), and this 24-ms singleton-presence benefit was significant, $t(19)=3.35, p=.003$. Error rates were similar on singleton present trials $(2.7 \%)$ and singleton absent trials $(2.8 \%), t(19)<1, p=.96$. Mean RT did not significantly differ as a function of the angular distance between the singleton and the target, $F(4,76)<$ $1, p=.473, \eta_{\mathrm{p}}{ }^{2}=.043$. If the color singleton captured covert spatial attention but was rejected before eye movements were initiated, we should have observed manual RT capture effects. These findings are inconsistent with rapid disengagement models.

\section{Overall saccadic reaction times}

This experiment was designed to produce faster saccadic latencies than observed in Experiment 2. Consistent with this, mean saccadic latencies (averaged across trial types) were indeed faster in this experiment (202 ms) than in Experiment 2 (225 ms), $t(37)=2.56, p=.014$. The mean saccade latencies in this experiment were also within the range observed in other additional singleton paradigm experiments (e.g., $251 \mathrm{~ms}$ in Wu \& Remington, 2003; $184 \mathrm{~ms}$ in Theeuwes et al., 2003, Experiment 2).

\section{First saccade destination}

The first saccade landing position results (see Fig. 6c) replicated those of Experiment 2. First saccades were much less likely to be directed toward the singleton distractor $(5 \%)$ than toward the average nonsingleton distractor $(12 \%), t(19)=$ 7.35, $p<.001$ (see the part of Fig. 6c labeled oculomotor suppression effect). The magnitude of this singleton suppression effect was nearly identical in Experiment $2(6 \%)$ and Experiment $3(7 \%), t(38)<1, p=.55$.

As in Experiment 2, we also analyzed the effect of singleton presence on first saccades to the target and nonsingleton distractors in a two-way ANOVA, excluding the singleton location. First saccades were significantly more likely to be directed to the target $(45 \%)$ than to the average nonsingleton distractor $(12 \%), F(1,19)=$ $38.233, p<.001, \eta_{\mathrm{p}}{ }^{2}=.668$. This $33 \%$ effect presumably reflects the attentional priority of the target. Note that this target enhancement effect was marginally smaller in this experiment $(33 \%)$ than in Experiment 2 $(45 \%), t(38)=1.85, p=.07$. This likely reflects a decreased preparedness for the upcoming search display in this experiment, due to the decreased duration of central fixation prior to the onset of the search array. The ANOVA also indicated that first saccades were slightly but significantly more likely to move toward both nonsingleton distractors and targets when the singleton was present $(29 \%)$ than when the singleton was absent $(28 \%), F(1,19)=5.568, p=.029, \eta_{\mathrm{p}}{ }^{2}=.227$. This is consistent with the singleton suppression account, because suppressing the singleton increases the opportunity for gaze to be directed to the nonsingleton locations when the singleton is present. Finally, the interaction of singleton presence and item type was not significant, $F(1,19)$ $<1, p=.827, \eta_{\mathrm{p}}^{2}=.003$.

\section{Suppression effects by singleton angular distance}

Heat maps (Fig. 6d) also replicated the pattern obtained in Experiment 2: Participants were generally less likely to shift gaze to a given location if it contained a singleton distractor than a nonsingleton distractor. Suppression effects were significant at each singleton angular distance $(p<.001$; see details in supplementary online materials).

\section{Saccadic latencies}

As in Experiments 1 and 2, saccadic latencies were shorter when participants first landed on the singleton distractor $(185 \mathrm{~ms})$ than on a nonsingleton distractor $(197 \mathrm{~ms}), t(19)=$ $2.53, p=.03$, or target $(216 \mathrm{~ms}), t(19)=6.23, p<.001$ (see Table 4). Note, however, that saccade latencies when participants first landed on the target were not significantly different when the singleton was present $(216 \mathrm{~ms})$ than when it was absent $(217 \mathrm{~ms}), t(19)<1, p=.74$. This finding is not consistent with the rapid reorienting hypothesis. 


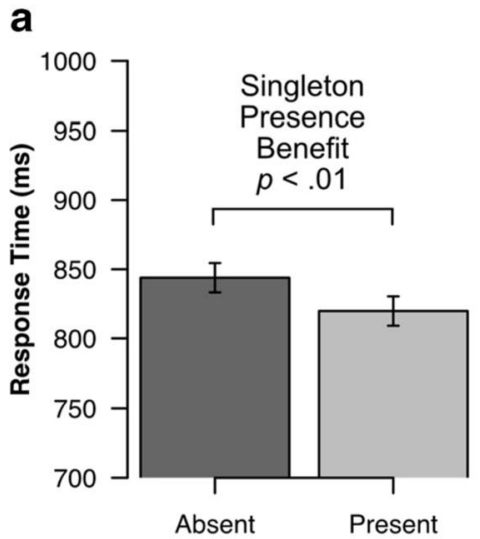

Singleton Presence

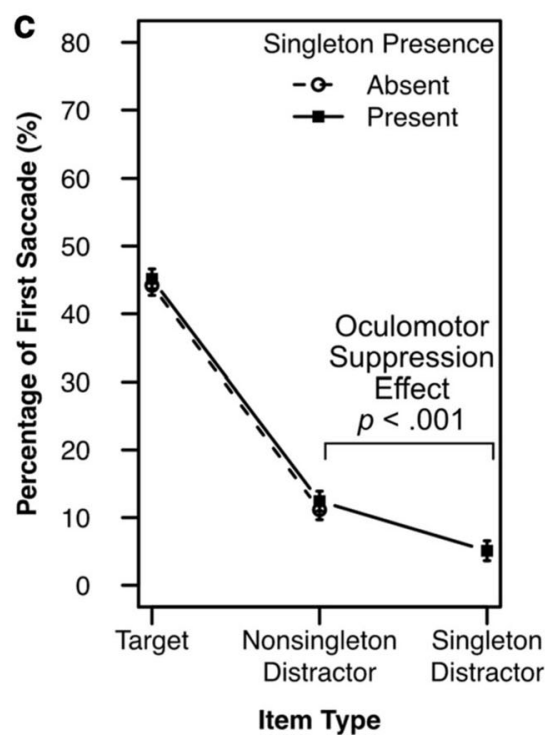

Fig. 6 Results from Experiment 3. (a) Manual response time as a function of singleton presence. (b) Manual response times as a function of the angular distance between the irrelevant singleton and the target. (c) Percentage of first saccades that landed on a given stimulus type (target, nonsingleton distractor, or singleton). (d) Heat maps of first saccade

\section{Suppression effects for the fastest quartile of saccadic latencies}

We conducted the same tests as in Experiment 2 to determine whether the overall pattern of results could be explained by an initial shift of covert attention to the singleton followed by rapid disengagement. First, we divided the data into quartiles on the basis of saccadic latency (see Table 5). As in Experiment 2, we observed significant suppression of gaze at the singleton location even in the fastest quartile (mean latency: $165 \mathrm{~ms}$ ), $t(19)=2.17, p$ $=.04$. Suppression effects were also significant in all other quartiles $(p<.001)$. Unlike Experiment 2 , the suppression effects were smaller in the fastest quartile (3\%) than in slowest quartile (7\%), and a one-way ANOVA yielded a significant main effect landing positon relative to the target, after rotating the data so that the target location appears at the top. Arrows point to the singleton location. See the online article for a color version of this figure. (Color figure online)

of quartile, $F(3,57)=5.41, p=.008, \eta_{\mathrm{p}}^{2}=.221$. As will be shown in next section, the fact that the oculomotor suppression effect was smallest in the fastest quartile indicates that these very rapid saccades were more likely to be random rather than controlled. Thus, the fact that there was still significant suppression and not enhancement of eye movements toward the singleton at the fastest quartile - in which the mean saccadic RT was $165 \mathrm{~ms}$ - is consistent with the signal suppression hypothesis and is inconsistent with the rapid disengagement hypothesis.

\section{Continuous gaze position}

We also examined gaze position at each sample period (see supplementary material for an animated plot of each angular 
Table 4 Mean saccadic latency (ms) by first saccade landing position for Experiment 3

\begin{tabular}{llll}
\hline & Target & Nonsingleton Distractor & Singleton Distractor \\
\hline Singleton Present & $216(20.6)$ & $197(14.9)$ & $185(26.3)$ \\
Singleton Absent & $217(23.1)$ & $195(16.8)$ & - \\
\hline
\end{tabular}

Note. Standard deviations are shown in parentheses.

distance). As shown in Fig. 7a, gaze position at the beginning of the trial was randomly distributed among the singleton, nonsingleton, and target locations. As the trial progressed, however, gaze position become more selective, with most samples located within the target region, fewer within the nonsingleton distractor regions, and even fewer within the singleton distractor region. Thus, in contrast with the prediction of the rapid disengagement hypothesis, gaze was not more likely to be directed toward the color singleton than toward the other locations early in the trial.

We performed the same formal analyses of these data as in Experiment 2. As shown in Fig. 7b, samples were equally likely to be in the target, singleton distractor, and nonsingleton distractor regions until approximately $200 \mathrm{~ms}$, and at no time point were samples more likely to occur in the singleton region than in the average of the nonsingleton distractor regions. Gaze samples were significantly more likely to be located within the average of the nonsingleton distractor regions than within the singleton region from $196 \mathrm{~ms}$ to $400 \mathrm{~ms}$ after the search array onset, which was a significant effect after using a permutation test to control the Type I error rate. This pattern of results provides no support for the hypothesis that gaze was captured by the singleton distractor early in the trial. Instead, the singleton location was rapidly suppressed.

\section{Discussion}

Experiment 3 replicated the results of Experiment 2 using methods that encouraged faster saccadic latencies to see if this caused the emergence of singleton capture. Saccadic latencies were indeed significantly faster in Experiment 3 than in Experiment 2, but first saccades to the task-irrelevant color singleton were still suppressed. Further analyses revealed that this suppression effect was not due to initial capture followed by rapid disengagement. These results support the signal suppression hypothesis and are inconsistent with purely bottom-up and top-down theories.

\section{General discussion}

A key debate in visual attention research is whether salient stimuli, such as color singletons, can automatically capture visual attention. Stimulus-driven theories propose that salient stimuli will be prioritized regardless of an observer's goals, whereas goal-driven theories propose that salient-butirrelevant stimuli have no special attention-attracting powers. In this study, we investigated a hybrid signal suppression hypothesis, which proposes that salient stimuli automatically generate a bottom-up "attend-to-me" signal, but that this signal can be actively suppressed by top-down control mechanisms to prevent the allocation of attention to a salient item. Previous research has shown some evidence of active suppression of covert attention in behavioral studies (Gaspelin et al., 2015) and electrophysiological studies (Gaspar \& McDonald, 2014; Sawaki \& Luck, 2010), but there has been little evidence that humans can actively suppress eye movements to salient-butirrelevant singletons below baseline levels of processing.

\section{Summary of findings}

Experiment 1 validated our general approach under conditions that encouraged capture by color singletons. The target itself was a singleton, which forced participants to establish an attentional set for singletons more broadly. We found that first saccades were more likely to be directed toward singleton distractors than toward the average nonsingleton distractor. This demonstrates that our paradigm is sensitive to

Table 5 Mean saccadic latency (ms), likelihood of initially fixating the nonsingleton and singleton distractors, and suppression effects for each saccadic latency quartile in Experiment 3

\begin{tabular}{lllrr}
\hline Quartile & Mean Saccadic Latency & Nonsingleton Distractor & Singleton Distractor & Suppression Effect \\
\hline Fastest & 165 & $14 \%$ & $11 \%$ & $3 \%$ \\
Fast & 190 & $14 \%$ & $4 \%$ & $10 \%$ \\
Slow & 211 & $12 \%$ & $3 \%$ & $9 \%$ \\
Slowest & 256 & $11 \%$ & $3 \%$ & $8 \%$
\end{tabular}

Note. Suppression effects were calculated as the percentage of saccades landing on the average nonsingleton distractor minus the percentage of saccades landing on the singleton distractor. 
a

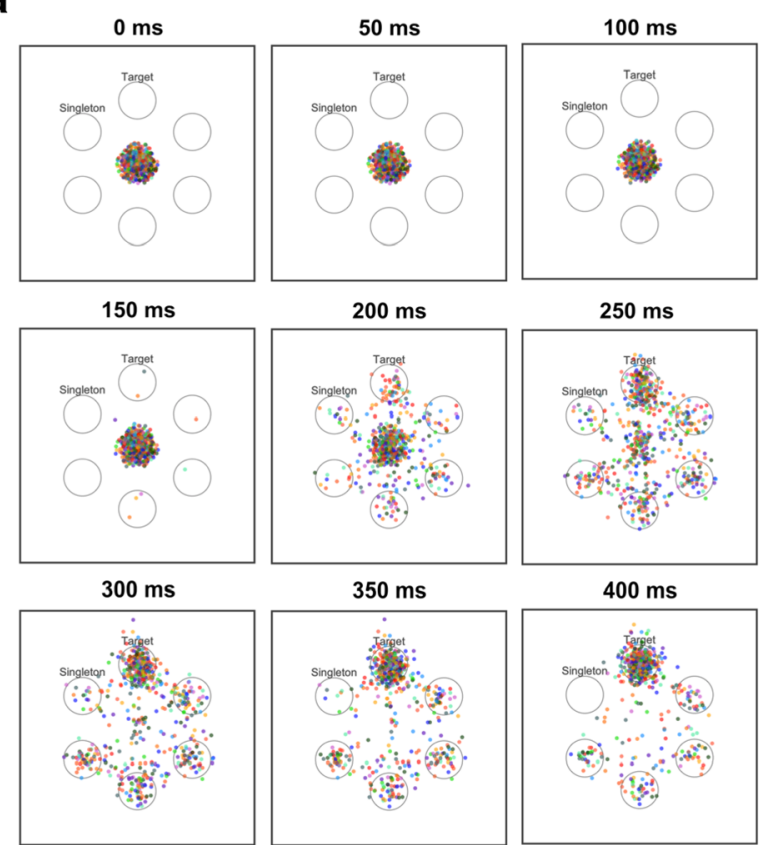

Fig. 7 Gaze position as a function of time relative to stimulus onset in Experiment 3. An animated plot can be found online as supplementary material. (a) Scatterplots of current gaze position at selected time points from trials in which the singleton was $60^{\circ}$ counterclockwise from the target (with the data rotated so that the target appears at the top

oculomotor capture effects and provides a valid method to investigate singleton suppression.

Experiment 2 discouraged singleton capture by using a target that was not a singleton shape and by holding the target shape constant throughout the entire session. Under these conditions, we found that first saccades were less likely to be directed toward singleton distractors than toward the average nonsingleton distractor-an oculomotor singleton suppression effect. Experiment 3 replicated the findings using modified parameters that encouraged faster saccadic latencies. Even though saccadic latencies were significantly faster, first saccades were still less likely to be directed to singleton distractors than to nonsingleton distractors.

\section{The rapid disengagement hypothesis}

Detailed analyses of the suppression effects in Experiments 2 and 3 demonstrated that the pattern of results could not be explained by adding the rapid disengagement hypothesis to the stimulus-driven account. First, if covert attention had been directed to the color singleton without an overt eye movement, we should have observed singleton-presence costs on manual RT. However, no evidence of singleton capture was found in the manual response data in Experiments 2 and 3. In fact, participants demonstrated a marginally significant singleton presence benefit in Experiment 2 and a significant singleton b

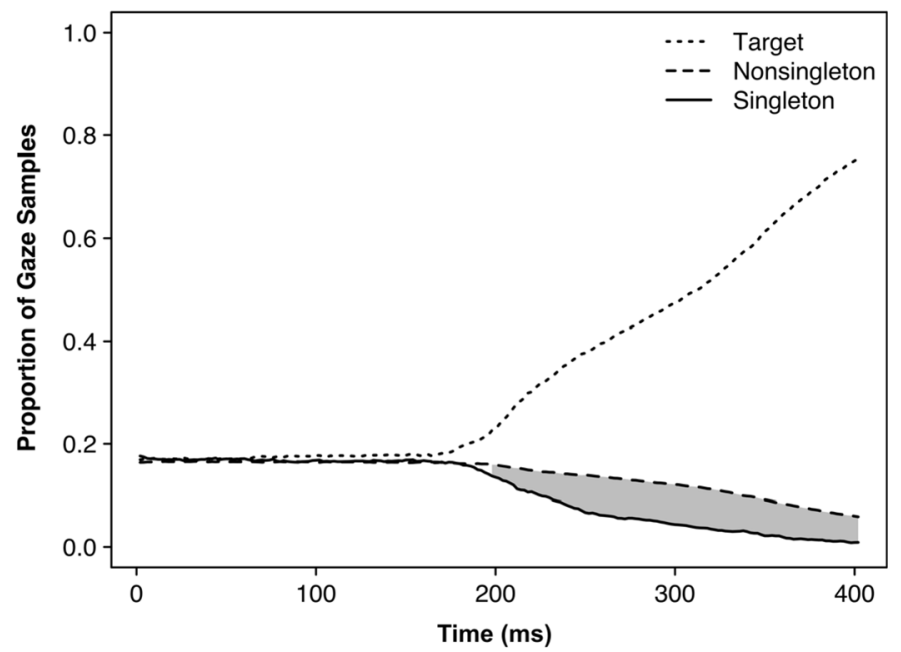

position). (b) Data from (a) reformatted as the proportion of gaze samples in the three object interest regions (target, singleton distractor, average nonsingleton distractor) at each time point. For simplicity, we averaged across every singleton angular distance. (Color figure online)

presence benefit in Experiment 3, suggesting that the color singleton did not capture covert attention but was actively suppressed, effectively reducing the relevant set size of object to be searched.

In addition, the rapid disengagement hypothesis predicts that color singletons should capture attention on trials with fast saccades. However, even when we constrained our analyses to the fastest quartile of saccades, first saccades were still less likely to land on singleton distractors than on nonsingleton distractors. In Experiment 3, singleton suppression was observed even in a quartile with a mean saccadic RT of only $165 \mathrm{~ms}$, demonstrating that suppression can occur very rapidly. Finally, we computed a continuous measure of gaze position at each sample point, ignoring saccades and fixations altogether, to see if gaze was more likely to be directed to the region of the singleton early in the trial. We found no evidence of capture by singletons in the first $200 \mathrm{~ms}$ of stimulus presentation, and after $200 \mathrm{~ms}$ singletons were suppressed. There was no time at which oculomotor capture was observed. In summary, although dwell times may sometimes play an important role in the size of observed capture effects (Fukuda \& Vogel, 2011; Gaspelin, Ruthruff, \& Lien, 2016; Geng \& Diquattro, 2010; Theeuwes, 2010; van Zoest et al., 2004), the current suppression effects cannot be attributed to mere rapid disengagement. 


\section{The attentional window hypothesis}

Another prominent modification of the stimulus-driven account is the attentional window hypothesis, which proposes that a salient stimulus will only capture attention when it appears inside the current attentional window (Belopolsky \& Theeuwes, 2010; Belopolsky, Zwaan, Theeuwes, Kramer, \& Ramer, 2007; Theeuwes, 2004; but see Gaspelin, Ruthruff, Lien, \& Jung, 2012). According to this hypothesis, participants will adopt a serial search strategy for difficult search tasks, and this will yield a small attentional window that does not include the salient singleton. If participants had adopted a serial search strategy in our feature search mode experiments, then this could explain a lack of capture in these experiments. However, this is not consistent with the overall pattern of results.

If participants had searched serially in the feature search mode experiments (Experiments 2 and 3), they should have had a much greater likelihood of fixating a nontarget item before fixating the target in these experiments than in the singleton detection mode experiment (Experiment 1). However, first saccades were approximately equally likely to be directed to the target under singleton-detection mode in Experiment $1(58 \%)$ and under feature-search mode in Experiment 2 (55\%), which both used identical stimulus timings. Moreover, if participants had made multiple shifts of covert attention before fixating the target under feature search mode, then this would have increased the saccadic latencies relative to singleton detection mode. However, mean saccade latencies for trials on which the first saccade landed on the target on singleton-absent trials were approximately equal in Experiments 1 and 2 (256 vs. $250 \mathrm{~ms}$, respectively; see Tables 1 and 2) and were even faster in Experiment 3 (217 ms; see Table 4). Thus, the results provided no evidence that participants engaged in serial search in Experiments 2 and 3. Overall RT did differ between search modes, but this may have resulted from a later stage of processing.

In general, most previous research that has used sophisticated approaches to distinguishing between parallel and serial processing has found that difficult feature search tasks involve parallel rather than serial processing; this research explains the increased RTs in difficult search tasks by means of limitedcapacity parallel search (Mordkoff \& Yantis, 1993; Townsend, 1971, 1976, 1990) or decision noise (Palmer, 1995; Palmer, Verghese, \& Pavel, 2000). Thus, the attentional window account will be plausible only for search tasks that clearly require serial search (see Woodman \& Luck, 2003). Moreover, even if the attentional window was more tightly focused under feature-search mode, this could explain an elimination of capture effects, but the attentional window account provides no means of explaining the observed singleton suppression effects in first saccades. Without an added suppressive mechanism, the attentional window account provides no reason to expect below-baseline processing of the color singleton.

\section{Potential mechanisms of suppression}

The current experiments suggest that salient feature singletons can be deprioritized during visual search, which we call a suppression effect (see also Gaspelin et al., 2015). Suppression effects can be explained by at least three possible mechanisms. First, participants may learn to suppress stimuli directly on the basis of their salience, which we call a singleton suppression mechanism. For example, Sawaki and Luck (2010, Experiment 4) varied target color and singleton color randomly trial by trial in a visual-search task. Even though participants had no foreknowledge of the upcoming singleton feature value, color singletons elicited an electrophysiological index of suppression, the $\mathrm{P}_{\mathrm{D}}$ component. This suggests that participants actively suppressed the singletons based on the fact that they were color singletons per se, without knowledge of the specific color of the singleton.

Second, participants may learn to suppress a specific feature value (e.g., such as red), which we call a featural suppression mechanism (Gaspar \& McDonald, 2014; Moher \& Egeth, 2012; Vatterott \& Vecera, 2012; Zehetleitner, Goschy, \& Müller, 2012). For example, Vatterott and Vecera (2012) had participants perform several blocks of an additional singleton paradigm, with a differently colored singleton on each block. Participants showed capture effects by the color singleton in the early portion of a given block, but the capture effects disappeared by the later portion of the block. This suggests that participants can learn to suppress a specific irrelevant feature value. Finally, participants may preattentively boost features that match the target (which would exclude the singleton), which is called featural upweighting (Bichot, Rossi, \& Desimone, 2005). Note that these accounts are not mutually exclusive - participants may adopt more than one of these strategies at a given moment, or they may adopt different strategies depending on experience and task demands. Future research on suppression in attentional capture should focus on testing between these alternative accounts.

As far as we know, most tests of the signal suppression hypothesis have focused solely on one type of salient stimuli: color singletons (e.g., Gaspar \& McDonald, 2014; Gaspelin et al., 2015; Jannati et al., 2013; Sawaki \& Luck, 2010; Vatterott \& Vecera, 2012). However, there is good reason to suspect that other types of dynamic salient stimuli, such as suddenly appearing stimuli (abrupt onsets), may be processed in a fundamentally different manner by the visual system. For example, several studies have shown that abrupt onsets capture attention under circumstances where color singletons do not capture attention (Franconeri \& Simons, 2003; Gaspelin et al., 2012; Jonides \& Yantis, 1988). Future research should test whether other types of salient stimuli, such as abrupt onsets, can be actively suppressed. 


\section{Conclusions}

In summary, the current results extend previous research on suppression of attentional capture to the field of oculomotor capture. The signal suppression hypothesis provides a more compelling account of previous results than either the strict stimulus-driven or goal-driven hypotheses alone. Future research will be needed to better understand the nature of suppressive attentional control and its temporal dynamics.

Acknowledgments This study was made possible by NIH Grants R01MH076226 and R01MH065034 to S.J.L. and postdoctoral fellowships T32EY013587 and F32EY024834 to N.G. from the National Eye Institute.

Author contributions N.G., C.J.L., and S.J.L. developed the study concept and design. Stimulus presentation programming and data analysis were performed by N.G. N.G. drafted the manuscript, and C.J.L. and S.J.L. provided editorial suggestions. All authors approved of the final version.

\section{References}

Awh, E., Belopolsky, A. V., \& Theeuwes, J. (2012). Top-down versus bottom-up attentional control: A failed theoretical dichotomy. Trends in Cognitive Sciences, 16(8), 437-443.

Bacon, W. F., \& Egeth, H. E. (1994). Overriding stimulus-driven attentional capture. Perception \& Psychophysics, 55(5), 485-496.

Belopolsky, A. V., \& Theeuwes, J. (2010). No capture outside the attentional window. Vision Research, 50(23), 2543-2550.

Belopolsky, A. V., Zwaan, L., Theeuwes, J., Kramer, A. F., \& Ramer, A. R. F. K. (2007). The size of an attentional window modulates attentional capture by color singletons. Psychonomic Bulletin \& Review, 14(5), 934-938.

Bichot, N. P., Rossi, A. F., \& Desimone, R. (2005). Parallel and serial neural mechanisms for visual search in macaque area V4. Science, 308(5721), 529-534.

Brainard, D. H. (1997). The psychophysics toolbox. Spatial Vision, 10(4), 433-436.

Burra, N., \& Kerzel, D. (2014). The distractor positivity (Pd) signals lowering of attentional priority: Evidence from event-related potentials and individual differences. Psychophysiology, 51, 685-696.

Chen, P., \& Mordkoff, J. T. (2007). Contingent capture at a very short SOA: Evidence against rapid disengagement. Visual Cognition, 15(6), 637-646.

Cosman, J. D., \& Vecera, S. P. (2013). Learned control over distraction is disrupted in amnesia. Psychological Science, 24(8), 1585-1590.

Eimer, M., \& Kiss, M. (2008). Involuntary attentional capture is determined by task set: Evidence from event-related brain potentials. Journal of Cognitive Neuroscience, 20(8), 1423-1433.

Fischer, B., \& Breitmeyer, B. (1987). Mechanisms of visual attention revealed by saccadic eye movements. Neuropsychologia, 25(1A), 73-83.

Folk, C. L., \& Remington, R. W. (1998). Selectivity in distraction by irrelevant featural singletons: Evidence for two forms of attentional capture. Journal of Experimental Psychology: Human Perception and Performance, 24(3), 847-858.

Folk, C. L., Remington, R. W., \& Johnston, J. C. (1992). Involuntary covert orienting is contingent on attentional control settings. Journal of Experimental Psychology: Human Perception and Performance, 18(4), 1030-1044.
Franconeri, S. L., \& Simons, D. J. (2003). Moving and looming stimuli capture attention. Perception \& Psychophysics, 65(7), 999-1010.

Fukuda, K., \& Vogel, E. K. (2011). Individual differences in recovery time from attentional capture. Psychological Science, 22(3), 361368.

Gaspar, J. M., Christie, G. J., Prime, D. J., Jolicoeur, P., \& McDonald, J. M. (2016). Inability to suppress salient distractors predicts low visual working memory capacity. Proceedings of the National Academy of Sciences of the United States of America, 113(13), 3693-3698.

Gaspar, J. M., \& McDonald, J. J. (2014). Suppression of salient objects prevents distraction in visual search. The Journal of Neuroscience, 34(16), 5658-5666.

Gaspelin, N., Leonard, C. J., \& Luck, S. J. (2015). Direct evidence for active suppression of salient-but-irrelevant sensory inputs. Psychological Science, 22(11), 1740-1750.

Gaspelin, N., Margett-Jordan, T., \& Ruthruff, E. (2014). Susceptible to distraction: Children lack top-down control over spatial attention capture. Psychonomic Bulletin \& Review, 22(2), 461-468.

Gaspelin, N., Ruthruff, E., \& Lien, M. (2016). The problem of latent attentional capture: Easy visual search conceals capture by taskirrelevant abrupt onsets. Journal of Experimental Psychology: Human Perception and Performance, 42(8), 1104-1120.

Gaspelin, N., Ruthruff, E., Lien, M.-C., \& Jung, K. (2012). Breaking through the attentional window: Capture by abrupt onsets versus color singletons. Attention, Perception, \& Psychophysics, 74(7), 1461-1474.

Geng, J. J., \& Diquattro, N. E. (2010). Attentional capture by a perceptually salient non-target facilitates target processing through inhibition and rapid rejection. Journal of Vision, 10(6), 5. doi:10.1167/10.6.5

Groppe, D., Urbach, T., \& Kutas, M. (2011). Mass univariate analysis of event-related brain potentials/fields: I. A critical tutorial review. Psychophysiology, 141(4), 520-529.

Henderson, J. M. (2003). Human gaze control during real-world scene perception. Trends in Cognitive Sciences, 7(11), 498-504. doi:10.1016/j.tics.2003.09.006

Hickey, C., Di Lollo, V., \& McDonald, J. J. (2009). Electrophysiological indices of target and distractor processing in visual search. Journal of Cognitive Neuroscience, 21(4), 760-775.

Hoffman, J. E., \& Subramaniam, B. (1995). The role of visual attention in saccadic eye movements. Perception \& Psychophysics, 57(6), 787795.

Hutton, S. B., \& Ettinger, U. (2006). The antisaccade tas as a research tool in psychopathology: A critical review. Psychophysiology, 43, 302313.

Ipata, A. E., Gee, A. L., Gottlieb, J., Bisley, J. W., \& Goldberg, M. E. (2006). LIP responses to a popout stimulus are reduced if it is overtly ignored. Nature Neuroscience, 9(8), 1071-1076. doi:10.1038/nn1734

Jannati, A., Gaspar, J. M., \& McDonald, J. J. (2013). Tracking target and distractor processing in fixed-feature visual search: Evidence from human electrophysiology. Journal of Experimental Psychology: Human Perception and Performance, 39(6), 1713-1730. doi:10.1037/a0032251

Jonides, J., \& Yantis, S. (1988). Uniqueness of abrupt visual onset in capturing attention. Perception \& Psychophysics, 43(4), 346-354.

Kim, M.-S., \& Cave, K. R. (1995). Spatial attention in visual search for features and feature conjunctions. Psychological Science, 6(6), 376380. doi:10.1111/j.1467-9280.1995.tb00529.x

Leber, A. B., \& Egeth, H. E. (2006). Attention on autopilot: Past experience and attentional set. Visual Cognition, 14(4/8), 565-583.

Leonard, C. J., \& Luck, S. J. (2011). The role of magnocellular signals in oculomotor attentional capture. Journal of Vision, 11(13), 1534 7362 .

Leonard, C. J., Robinson, B. M., Hahn, B., Gold, J. M., \& Luck, S. J. (2014). Enhanced distraction by magnocellular salience signals in schizophrenia. Neuropsychologia, 56, 359-366. 
Loftus, G. R., \& Masson, M. E. J. (1994). Using confidence intervals in within-subject designs. Psychonomic Bulletin \& Review, 1(4), 476490.

Maris, E., \& Oostenveld, R. (2007). Nonparametric statistical testing of EEG- and MEG-data. Journal of Neuroscience Methods, 164(1), 177-190.

Moher, J., \& Egeth, H. E. (2012). The ignoring paradox: Cueing distractor features leads first to selection, then to inhibition of tobe-ignored items. Attention, Perception, \& Psychophysics, 74(8), $1590-1605$

Moher, J., Lakshmanan, B. M., Egeth, H. E., \& Ewen, J. B. (2014). Inhibition drives early feature-based attention. Psychological Science, 25(2), 315-324.

Mordkoff, J. T., \& Yantis, S. (1993). Dividing attention between color and shape: Evidence of coactivation. Perception \& Psychophysics, 53(4), 357-366.

Mounts, J. R. W. (2000). Attentional capture by abrupt onsets and feature singletons produces inhibitory surrounds. Perception \& Psychophysics, 62(7), 1485-1493.

Oakes, L. M., Baumgartner, H. A., Barrett, F. S., Messenger, I. M., \& Luck, S. J. (2013). Developmental changes in visual short-term memory in infancy: Evidence from eye-tracking. Frontiers in Psychology, 4(Oct.), 1-13.

Palmer, J. (1995). Attention in visual search: Distinguishing four causes of a set-size effect. Current Directions in Psychological Science, 4(4), 118-123.

Palmer, J., Verghese, P., \& Pavel, M. (2000). The psychophysics of visual search. Vision Research, 40(10/12), 1227-1268.

Reuter-Lorenz, P. A., Hughes, H. C., \& Fendrich, R. (1991). The reduction of saccadic latency by prior offset of the fixation point: An analysis of the gap effect. Perception \& Psychophysics, 49(2), $167-175$.

Sawaki, R., \& Luck, S. J. (2010). Capture versus suppression of attention by salient singletons: Electrophysiological evidence for an automatic attend-to-me signal. Attention, Perception, \& Psychophysics, 72(6), 1455-1470.

Sawaki, R., \& Luck, S. J. (2011). Active suppression of distractors that match the contents of visual working memory. Visual Cognition, 19(7), 956-972.

Theeuwes, J. (1992). Perceptual selectivity for color and form. Perception \& Psychophysics, 51(6), 599-606.

Theeuwes, J. (2004). Top-down search strategies cannot override attentional capture. Psychonomic Bulletin \& Review, 11(1), 65-70.

Theeuwes, J. (2010). Top-down and bottom-up control of visual selection. Acta Psychologica, 135(2), 77-99.
Theeuwes, J., Atchley, P., \& Kramer, A. F. (2000). On the time course of top-down and bottom-up control of visual attention. Control of Cognitive Processes: Attention and Performance, XVIII, 105-124.

Theeuwes, J., De Vries, G.-J., \& Godijn, R. (2003). Attentional and oculomotor capture with static singletons. Perception \& Psychophysics, 65(5), 735-746.

Theeuwes, J., Kramer, A. F., Hahn, S., \& Irwin, D. E. (1998). Our eyes do not always go where we want them to go: Capture of the eyes by new objects. Psychological Science, 9(5), 379-385.

Theeuwes, J., Kramer, A. F., Hahn, S., Irwin, D. E., \& Zelinsky, G. J. (1999). Influence of attentional capture on oculomotor control. Journal of Experimental Psychology: Human Perception and Performance, 25(6), 1595-1608.

Townsend, J. T. (1971). A note on the identifiability of parallel and serial processes. Perception \& Psychophysics, 10(3), 161-163.

Townsend, J. T. (1976). Serial and within-stage independent parallel model equivalence on the minimum completion time. Journal of Mathematical Psychology, 14(3), 219-238.

Townsend, J. T. (1990). Serial vs. parallel processing: Sometimes they look like Tweedledum and Tweedledee but they can (and should) be distinguished. Psychological Science, 1(1), 46-54.

van Zoest, W., Donk, M., \& Theeuwes, J. (2004). The role of stimulusdriven and goal-driven control in saccadic visual selection. Journal of Experimental Psychology: Human Perception and Performance, 30(4), 746-759.

Vatterott, D. B., \& Vecera, S. P. (2012). Experience-dependent attentional tuning of distractor rejection. Psychonomic Bulletin \& Review, 19(5), 871-878

Wolfe, J. M. (2007). Guided Search 4.0: Current progress with a model of visual search. In W. D. Gray (Ed.), Integrated models of cognitive systems (pp. 99-119). New York, NY: Oxford University Press.

Wolfe, J. M., Cave, K. R., \& Franzel, S. L. (1989). Guided Search: An alternative to the feature integration model for visual search. Journal of Experimental Psychology: Human Perception and Performance, 15(3), 419-433.

Woodman, G. F., \& Luck, S. J. (2003). Serial deployment of attention during visual search. Journal of Experimental Psychology. Human Perception and Performance, 29(1), 121-138.

Wu, S., \& Remington, R. W. (2003). Characteristics of covert and overt visual orienting: Evidence from attentional and oculomotor capture. Journal of Experimental Psychology: Human Perception and Performance, 29(5), 1050-1067.

Zehetleitner, M., Goschy, H., \& Müller, H. J. (2012). Top-down control of attention: It's gradual, practice-dependent, and hierarchically organized. Journal of Experimental Psychology: Human Perception and Performance, 38(4), 941-957. 